\title{
Analysis on the Strategy of the Development of Mongolian Medicine Brand
}

\author{
Huilin $\mathrm{An}^{1 *}$, Yun $\mathrm{Li}^{1}$, Zhiwei Zhang ${ }^{1}$ and Wenhui Fan ${ }^{1}$

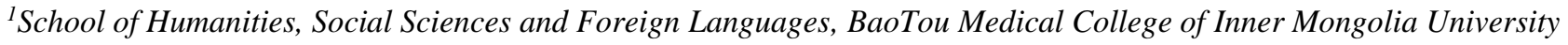 \\ of Science \& Technology, Baotou, Inner Mongolia, 014060, China \\ *Corresponding author. Email: 102013204@btmc.edu.cn
}

\begin{abstract}
With the support of national and regional policies, Mongolian medicine enterprises keep a good development momentum, but there is a certain gap with the leading Chinese ethnic minority medicine. Some products of Mongolian medicine, to some extent, have been recognized, but the overall level and universality still need to be improved. This paper analyzes the current overall development of Mongolian medicine from the perspective of a service coordinated system, the integration of regional industry and the development of the industry. In addition, this paper also analyzes problems in its current development from the perspective of brand development strategy, brand awareness, brand building, brand development and brand extension and puts forward the corresponding countermeasures and suggestions. This paper argues that the development of the Mongolian medicine brand is still in its initial stage and there is a long way to revitalize the Mongolian medicine industry.
\end{abstract}

Keywords: Mongolian medicine; Mongolian medicine enterprises; brand strategy; Chinese ethnic minority

medicine

\section{INTRODUCTION}

Chinese ethnic minority medicine is the treasure of traditional Chinese medicine culture, and Mongolian medicine is one of the important branches of ethnic minority medicine. The sustainable development of Mongolian medicine is inseparable from the guarantee and supply of Mongolian medicine. Mongolian medicine not only pays attention to conquering difficult diseases but also involves the concept of health care and treatment methods by Mongolian medicine. In the clinical treatment of the fight against COVID-19, the intervention of integrated traditional Chinese and western medicine has achieved remarkable results. During this period, some hospitals in Inner Mongolia adopted Mongolian medicine to intervene in the treatment of COVID-19, which also had certain effects on the treatment of COVID-19. Although Mongolian medicine plays an irreplaceable role in the treatment of diseases and the development of traditional medicine, Mongolian medicine has a long way to go before it can stand out and achieve long-term development in the context of fierce competition in the pharmaceutical market in view of its development process and as far as is concerned the mature market of Western medicine and the challenges of the relatively rapid development of other ethnic minority medicine.

\section{ANALYSIS OF THE DEVELOPMENT STATUS OF MONGOLIAN MEDICINE}

\subsection{It has formed a relatively perfect coordinated system of Mongolian medicine inheritance, development and service}

Mongolian medicine begins in the Ming Dynasty, flourishes in the Qing Dynasty and blooms in modern time. There are 123 Mongolian hospitals (including traditional Chinese medicine hospitals and Mongolian and Tibetan medicine hospitals) in China, among which there are 18 level-III hospitals and 89 hospitals of level-II mainly located in the Inner Mongolia Autonomous Region and Mongol communities in some other provinces and regions. There are 124 enterprises related to Mongolian medicine, 112 in Inner Mongolia and 12 outside the Autonomous Region, among which 6 enterprises are specialized in Mongolian medicine. There are 12 Mongolian medicine societies, among which there are 8 universities (colleges) having a major related to Mongolian medicine, 29 research institutes of Mongolian medicine and 5 Mongolian medicine museums[1]. The Inner Mongolia Autonomous Region has established the Administration of Mongolian Traditional Medicine and has set up branches in its 12 affiliated cities. Tongliao, the capital of Mongolian medicine in China, has set up a special development fund and social organizations such as Tongliao Association for 
the Promotion of Scientific Development of Mongolian Medicine Industry to jointly promote the rapid, healthy and sustainable development of Mongolian medicine.

\subsection{It should expand Mongolian medicine enterprises and form regional industrial integration}

We searched enterprises related to Mongolian medicine in Qichacha professional edition and identified 124 Mongolian medicine enterprises according to names and scope of business, among which there are 112 enterprises in Inner Mongolia, 1 in Liaoning province, 1 in Beijing, 3 in Qinghai province, 5 in Xinjiang Uygur Autonomous Region and 2 in Sichuan province. In terms of business scope, there are 40 enterprises involving in the planting of Mongolian herbs, 38 enterprises researching and developing Mongolian medicine, 39 enterprises producing Mongolian medicine, and 84 enterprises selling Mongolian medicine, all of which involve in vertically integrated enterprises of Mongolian medicine[1]. However, in order to facilitate enterprises to engage in production and operation, many natural person proprietorship enterprises have registered a relatively broad business scope in the marketing license; but in fact, they are not fully capable of large-scale cultivation, research and development, production or sales of Mongolian medicine. For example, in terms of Mongolian medicine cultivation, there are only 6 enterprises specialized in the planting of Mongolian herbs, which are established late with small scale. However these planting enterprises form a large-scale industrial belt for the planting of Mongolian herbs, which to some extent improves the self-sufficiency rate of its raw material in the region and realizes its economies of scope.

Table 1. The main indexes of pharmaceutical manufacturing in the regions of ethnic minority medicine in 2017

\begin{tabular}{|c|c|c|c|c|c|}
\hline Region & Nation & Inner Mongolia & Guizhou & Tibet +Qinghai & Xinjiang \\
\hline $\begin{array}{c}\text { Industrial sales output (100 } \\
\text { million) }\end{array}$ & 28417.72 & 313.62 & 391.29 & 75.64 & 38.16 \\
\hline $\begin{array}{c}\text { Growth rate of industrial } \\
\text { sales output }\end{array}$ & $10.41 \%$ & $-0.92 \%$ & $11.37 \%$ & $0.49 \%$ & $28.66 \%$ \\
\hline Total assets (100 million) & 28789.11 & 363.22 & 441.09 & 119.8 & 144.54 \\
\hline Growth rate of total assets & $14.83 \%$ & $12.94 \%$ & $18.49 \%$ & $6.36 \%$ & $11.94 \%$ \\
\hline
\end{tabular}

Data resources: Industrial sales output and total assets are from 2017 China Industry Statistics Yearbook, the growth rate of industrial sales output and growth rate of total assets are calculated automatically by the 2017 China Industry Statistics Yearbook and 2016 China Industry Statistics Yearbook

\subsection{Mongolian medicine has a good development momentum, but there is still a gap with the well-developed ethnic minority medicine}

In the process of long-term development, ethnic minority medicine has experienced different conditions, and few ethnic minority medicines eventually form a relatively complete medical system. As an indispensable part in the development of ethnic minority medicine, ethnic minority medicine has a direct impact on the survival of Chinese medicine. Inner Mongolian Medicine Co., LTD., Inner Mongolia Kulun Mongolian Medicine Factory and other Mongolian medicine enterprises have established Mongolian medicine brands such as Mongolian King and Anyou. Mongolian medicine, such as Erridun - Urirl (Zhenbao pill), Poly and Zachong 13 herbs, are relatively popular. The annual capacity of the major Mongolian medicine enterprises exceeds 2,000 tons [2]. Inner Mongolia, Guizhou, Tibet, Qinghai and Xinjiang are the major places of ethnic minority medicine of Mongolian medicine, Miao medicine, Tibetan medicine and Uighur medicine. Compared with the main indexes of pharmaceutical manufacturing in these areas (see table 1), it can be seen that main economic indicators of pharmaceutical manufacturing in the Inner Mongolia autonomous region rank only second to those in Guizhou pharmaceutical manufacturing, but far beyond in Tibet, Qinghai and Xinjiang region. However, in terms of the growth rate, the total assets of the pharmaceutical manufacturing industry in Inner Mongolia continue to grow, but the growth of industrial sales output is relatively slow. Although the total output value of the pharmaceutical manufacturing industry in Inner Mongolia is relatively high, the annual output value of Mongolian medicine is lower than that of Tibetan medicine and Miao medicine. The scale of Mongolia medicine enterprises is relatively small, unlike those of Miao Medicine and Tibetan medicine which have listed companies. Compared with other ethnic minority medicine, there is still a gap between Mongolian medicine industries and other ethnic minority medicine industries.

\section{PROBLEMS IN THE DEVELOPMENT OF MONGOLIAN MEDICINE BRAND}

\subsection{Poor brand awareness of Mongolian medicine enterprises and varied quality of Mongolian medicine products}

The number of Mongolian medicine enterprises is small, so does their scale, but they have a certain influence in certain areas. Compared with other traditional medicine and ethnic 
minority medicine, Inner Mongolia does not have national medicine brand and related products ranked in the top, nor listed Mongolian medicine enterprises. Its product scale and degree of modernization do not enjoy sufficient development. Although some Mongolian medicine products in Inner Mongolia have a high popularity and recognition among local Mongolian patients, patients have a low awareness of Mongolian medicine and have limited purchasing power. Although nearly 40 enterprises are producing Mongolian medicine, the whole production technology level is low. Few Mongolian medicines have been included in the national standard of medicinal materials and the national essential drugs and the catalogue of medical insurance.

\subsection{Homogeneous Mongolian medicine products and poor brand recognition}

Although there are a large number of Mongolian medicines, their names and structures are similar and undiversified. There are many kinds of Mongolian medicine with more than 2,200 kinds, and there are more than 1,300 kinds of commonly used medicines [3]. It has more than 6000 registered number of approvals for Mongolian medicine preparation, 128 varieties of Mongolian medicine with national medicine permission number, and 12 dosage forms including pill, powder, tablet, capsule and granule [4]. Although there are many varieties, their names and structures are similar and undiversified, such as Nuangong 7 herbs pills, Qingre 8 herbs powder, Jianwei 10 herbs pills, Honghuaqinggan 13 herbs pills, which are all named after their efficacy and the number of component medicines. In addition, some Mongolian medicines, such as Chagedri, Erridun-Urirl (Zhenbao Pill), Zhachong 13 herbs pill, Hardun Helumule 13 herbs pill and Xieriga 413 herbs tang powder, are directly named according to the Mongolian names or their Mongolian names and the number of component medicines. Consumers cannot be impressed by such similar naming methods. Especially for consumers who are from other ethnic groups other than the Mongol nationality, it is hard for them to remember awkward-sounding Mongolian names and thus buy medicines again. The packaging of some Mongolian medicines is backward, and those produced by small workshops even lack labels on relevant instructions, dates, ingredients or possible adverse reactions.

\subsection{Weak ability in the market development of Mongolian medicine products and lack of professional marketing personnel}

Most Mongolian medicine enterprises are weak in market development and management and few enterprises take advantage of Internet plus. There are few websites related to Mongolian medicine and some websites have the introduction of Mongolian medicine, but people cannot directly pay for the purchase, which, to some extent, limits consumers' desire to buy. Due to the weak capacity in marketing and brand building, in channel building, market research and other market development work, insufficient publicity of the pharmacology, efficacy and scope of effects of Mongolian medicine, and little understanding of the efficacy and its other aspects by people outside the Mongolian community, the popularity of Mongolian medicine does not spread among consumers [5]. Three medical colleges at the undergraduate level in Inner Mongolia have set up marketing majors, including the major of Mongolian medicine in two colleges and the major of Mongolian medicine and pharmacy in one. However, there are no relevant courses about Mongolian medicine in the training programs of these marketing majors. The professional medical marketing team lacks the knowledge of Mongolian medicine, which has a certain negative effect on the construction of high-quality marketing team of Mongolian medicine enterprises.

\subsection{Lack of the brand innovation consciousness of Mongolian medicine and its coordinated development}

Mongolian medicine enterprises are small in scale and weak in competitiveness with few R\&D investments. Mongolian medicine brands have not been formed and its market share is small. There are few Mongolian medical research institutes and most of them are attached to the institutions of Mongolian hospitals. The insufficient innovative research on Mongolian medicine and the coordination among institutions of high learning, governments and enterprises and incomplete modern pharmaceutical enterprise mechanism characterized by the integration of research, production and marketing limit the enterprises to innovate Mongolian medicine brands. The relatively long service cycle of Mongolian medicine is also affected by the lack of modernization on traditional medical methods, medical devices and Mongolian medicine. There is a shortage of scientific research talents and high-level talents in Mongolian medicine. Most of the high-end talents in Mongolian medicine are concentrated in the hospitals and research institutes of Mongolian medicine. There is a shortage of R\&D talents in Mongolian medicine enterprises There are 123 Mongolian medicine hospitals (including traditional Chinese medicine hospitals and Mongolian medicine and Tibetan medicine hospitals) in China. Currently, there are 124 enterprises operating in China, including Mongolian medicine, but only 39 enterprises produce Mongolian medicine and even fewer of them have the capacity of production. Such imbalance in the development of Mongolian medicine is not conducive to its long-term sustainable and healthy development. Mongolian hospitals are generally equipped with special Mongolian medicine preparation rooms, which also erect barriers for Mongolian medicine to enter Mongolian hospitals. 


\section{COUNTERMEASURES ON THE DEVELOPMENT OF MONGOLIAN MEDICINE BRAND}

\subsection{Establishing brand awareness for Mongolian medicine enterprises to improve their quality}

In addition to closely combine traditional culture with corporate philosophy, Mongolian medicine enterprises should strengthen their brand awareness and make a comprehensive plan for their brand. It should design and integrate the brand of Mongolian medicine products from the whole process of the value chain in order to strengthen patients' acknowledgment of the brand of Mongolian medicine and understand its characteristics. Good wine needs no bush. Brand cannot develop without its quality. Therefore, it should improve the standardization of Mongolian medicine and realize the quality management of its whole process. We should improve the quality of raw materials, strengthen our technique in the cultivation of Mongolian herbs, establish the quality standard, build regular planting base, realize its industrialization and ensure the reliable source of Mongolian herbs. In the production and processing stage of Mongolian medicines, it should implement relevant laws and regulations such as national drug production and drug standards to make sure that its quality is stable and controllable, thus carrying out the certification of quality system to make the process more standardized. It should actively strive to bring more Mongolian medicine products into the pharmaceutical catalogue and reduce the purchase cost of patients.

\subsection{Rebuilding and upgrading brands and create differentiated Mongolian medicine products}

Some Mongolian medicine enterprises with the ability of pharmaceutical research and development and innovation carry out brand rebranding and upgrading for their competitive products. Mongolian Administration of Traditional Chinese Medicine can manage the application of Mongolian medicine logo with Mongolian traditional culture and feature. Medicines that have registered number of approval of Mongolian preparation should label Mongolian logo in their packages. Mongolian medicine enterprises should mark their names and instruction in Chinese, which can not only inherit the Mongolian medicine intangible cultural heritage and the traditional culture, but also make Mongolian medicine popular among more patients. It should improve Mongolian medicine products from the aspects of the name, package, instructions for use, dosage form, etc., enhance the universality of Mongolian medicine cultural connotation, theory and cultural technology [6], and conform to the taste of the mass market. We can highlight the curative effect, enterprise brand and product brand of Mongolian medicines in the names, which can complement each other. It should keep pace with the times in its packaging design and improvement. The instructions should be standardized, easy to understand, which can be explained in Mongolian and Chinese to facilitate its use of a wider range of users.

\subsection{Expanding brand marketing channels of Mongolian medicine and build a professional team for the promotion of Mongolian medicine}

While ensuring the quality of Mongolian medicine, we should reduce the cost of each link in the value chain to leave a certain profit margin for middlemen and retailers. We should have such enterprises as having planting bases of Mongolian herbs, build modern ecological planting bases, provide diversified Mongolian medicine products and accessories, attract patients by combining Mongolian medicine ecotourism with health care experience of Mongolian medicine and enable more consumers to understand the connotation and characteristics of Mongolian medicine. We should strengthen the cooperation with Mongolian medical institutions and make an explanation and promotion of products and introduce its latest clinical trial on an irregular basis. We should build a Mongolian medicine counter in chain drugstores, popularize its health knowledge on the basis of drugstores and the community as the promotion unit, and solidified the consumer group. We should promote Mongolian medicine products on a regular basis through academic conferences, meetings, product training, strategic alliance and other forms and boost the development of the "Healthy Silk Road" through cultural exchanges of Mongolian medicine in Mongolia and other countries. Colleges and universities with majors related to Mongolian medicine and marketing should have courses related to Introduction of Mongolian Medicine in their training program for marketing majors, so as to enhance the knowledge of students majoring in marketing, expand their employment channels, and provide reserves for the specialization of marketing teams of Mongolian medicine enterprises. After being employed, we should strengthen their training and assessment they should strengthen the training and assessment on product knowledge and marketing methods and other professional knowledge to build lasting learning ability and improve the professional quality of marketing personnel.

\subsection{Enhancing the core competitiveness of Mongolian medicine enterprises and building a lasting innovation mechanism for brand extension}

By integrating the existing resources of Mongolian medicine enterprises, relying on the core Mongolian medicine enterprises and through mergers and strategic alliances, we should build the industry clusters of 
Mongolian medicine and regional integration to form its economies of scale economy. With the help of the government or the core enterprises, the existing production bases of Mongolian medicine should be integrated to establish a centralized production base of Mongolian medicine, so as to realize complementary advantages and optimize the allocation of resources, improve the current situation of small scale production and repeated production of various varieties of Mongolian medicine, implement the same medicine price, and reduce the internal cost resulted by the price war. Mongolian medicine enterprises have increased investment in research and development, actively participated in GMP certification of new drugs, strengthened cooperation between Mongolian medicine research institutes and institutions of high learning, participated in the development of new Mongolian medicine products, improved their innovation ability and modernization in Mongolian medicine, and also paid attention to the development of functional food and beverage of Mongolian medicine. We should promote both internal and external communication and in-depth interaction and integration of elements resources of the Mongolian medicine industry, increase the interaction among the government, enterprises, universities, research institutes and hospitals, and promote the in-depth integration of its production, education, research and application. In addition, we should strengthen the cooperation between Mongolian hospitals and Mongolian medicine enterprises, and balance the use of products between the preparation rooms of Mongolian medicine in hospitals and Mongolian medicine enterprises. What's more, we should increase investment in introducing high-level scientific research talents in Mongolian medicine enterprises, promote innovation in Mongolian medicine, and vigorously promote preparations, standardization and academic inheritance in Mongolian medicine hospitals.

\section{CONCLUSION}

With the support of the state for the development of ethnic minority medicine, Inner Mongolia has constantly introduced policies and specific measures to promote the continuous development and growth of the Mongolian medicine industry and the continuous improvement of its inheritance, development and service cooperation system. However, in its development, we find that there are some problems such as poor brand awareness of Mongolian medicine enterprises and varied quality of Mongolian medicine products; serious homogeneity and poor brand recognition; weak ability in the market development for Mongolian medicine products and lack of professional marketing personnel; lack of the brand innovation consciousness of Mongolian medicine and its coordinated development. In order to promote the development of
Mongolian medicine brand, it is suggested that it should establish brand awareness for Mongolian medicine enterprises to improve its quality, rebuild and upgrade brands and create differentiated Mongolian medicine products, expand brand marketing channels of Mongolian medicine and build a professional team for the promotion of Mongolian medicine and build a lasting innovation mechanism for brand extension, thus improving the core competitiveness of Mongolian medicine enterprises.

\section{ACKNOWLEDGMENT}

This paper is the phased achievement of Science Research Fund of Baotou Medical College "The Development of the Industry of Green Mongolian Medicine in Inner Mongolia" (BYJJ-SZ 201902) as well as the phased achievement of Baotou Young Talents Innovative Plan "Research on the Development Path of Baotou Mongolian Medicine Green Industry".

\section{REFERENCES}

[1] Zhang Zhiwei, Li Yun, An Huilin, Fan Wenhui. Green Mongolian Medicine[M]. Tianjin Science and Technology Press, 2019.8.

[2] Sun Yingli, Gong Fanli. Industry Environment analysis and Implementation Countermeasures for the Brand Development of Inner Mongolia Mongolian pharmaceutical Enterprises[J]. Journal of Inner Mongolia University for the Nationalities(Social Sciences), 2018.44(4):115-119.

[3] Celimuge, Song Lin, et al. Ideas on the Characteristics and Development of Mongolian Medicine $[\mathrm{J}]$. Chinese Journal of Library and Information Science for Traditional Chinese Medicine. 2016.(6):4-9.

[4] Wang Yujie. The Strategic Concept of "One Belt One Road", Reinforcing the Dream of Mongolian Medicine - the Development Trend of Mongolian Medicine Industrialization [J] Journal of North Pharmacy, 2015.(12):131-132.

[5] Wu Ritu, Wu Yunga. Thoughts on the Development Strategy of Inner Mongolia Mongolian Medicine Industry [J]. Journal of Minzu University of China (Nature and Science). 2013.22(8):56-61.

[6] Kong Jianjun, Chang Liang, On the Development of Grassroots Mongolian Medical Institutions: SWOT Analysis [J]. Journal of Medicine \& Pharmacy of Chinese Minorities. 2018.24(8):67-69. 